\title{
Le politique dans la cartographie. Tracé des frontières, carte et territoire lors du Congrès de Berlin en 1878
}

\section{Ute Schneider}

\section{OpenEdition}

\section{Journals}

Édition électronique

URL : http://journals.openedition.org/rh19/4449

DOl : $10.4000 /$ rh 19.4449

ISSN : $1777-5329$

\section{Éditeur}

La Société de 1848

\section{Édition imprimée}

Date de publication : 1 juin 2013

Pagination : 119-135

ISSN : 1265-1354

\section{Référence électronique}

Ute Schneider, « Le politique dans la cartographie. Tracé des frontières, carte et territoire lors du Congrès de Berlin en 1878 », Revue d'histoire du XIXe siècle [En ligne], 46 | 2013, mis en ligne le 01 juin 2016, consulté le 19 avril 2019. URL : http://journals.openedition.org/rh19/4449 ; DOI : 10.4000/ rh19.4449 


\section{UTE SCHNEIDER}

\section{Le politique dans la cartographie. Tracé des frontières, carte et territoire lors du Congrès de Berlin en 1878*}

Dans son analyse détaillée du troisième volume de l'histoire de la société allemande de Hans-Ulrich Wehler, Richard J. Evans reprochait aux historiens allemands «leur manque flagrant d'imagination visuelle [...] par rapport à leurs confrères français ${ }^{1}$. Cette affirmation célèbre a souvent été citée par la suite. La critique d'Evans faisait référence à l'absence totale de matériel cartographique dans l'histoire de la société allemande, qui privait de représentation l'espace de l'Histoire. Le fait qu'Evans eût attendu la parution $\mathrm{du}$ troisième volume pour formuler ce souhait tenait sans doute moins à la nécessité urgente de spatialiser l'histoire allemande de la deuxième moitié du XIX e siècle qu'au "retour de l'espace» qui se profilait dans les sciences humaines et sociales ${ }^{2}$. Ce tournant spatial ne devait pas épargner les sciences historiques allemandes. Depuis lors, de nombreuses études attentives à l'espace ont été publiées, prouvant que les historiens allemands ne craignaient plus les représentations graphiques ${ }^{3}$. Des manuels récents insistent désormais sur les «sentiments d'ancrage " ${ }^{4}$; les "frontières» se sont invitées dans les lieux de mémoire européens ${ }^{5}$, et, à l'échelle mondiale, Jürgen Osterhammel a dernièrement réaffirmé l'importance des espaces et des frontières ${ }^{6}$.

\footnotetext{
* Je remercie Marianne Floc'h pour sa traduction méticuleuse, Matthias Wischnewsky pour son soutien durant les recherches et Sonja Hillerich pour ses corrections avisées.

1. Richard J. Evans, Die Zeit, 13 octobre 1995, n 42.

2. Jürgen Osterhammel, «Die Wiederkehr des Raumes: Geopolitik, Geohistorie und historische Geographie", Neue Politische Literatur, tome 43 (1998), p. 374-397.

3. Citons par exemple Ingrid Baumgärtner, Paul-Gerhard Klumbies et Franziska Sick [dir.], Raumkonzepte. Disziplinäre Zugänge, Göttingen, V\&R Unipress, 2009; Stephan Günzel [dir.], Raum. Ein interdisziplinäres Handbuch, Stuttgart, Metzler, 2010; Holger Köhn, Die Lage der Lager. Displaced Persons-Lager in der amerikanischen Besatzungszone Deutschlands, Essen, Klartext, 2012; Iris Schröder, Das Wissen von der ganzen Welt. Globale Geographien und räumliche Ordnungen Afrikas und Europas 17901870, Paderborn, Ferdinand Schöningh, 2011.

4. Celia Applegate, «Senses Of Place», in Helmut Walser Smith [dir.], The Oxford Handbook of Modern German History, Oxford, Oxford University Press, 2011, p. 49-70.

5. Petra Deger, "Grenzen», in Pim den Boer, Heinz Duchhardt, Georg Kreis et Wolfgang Schmale [dir.], Europäische Erinnerungsorte. Mythen und Grundbegriffe des europäischen Selbstverständnisses, München, Oldenbourg, 2012, p. 247-256.

6. Jürgen Osterhammel, Die Verwandlung der Welt. Eine Geschichte des 19. Jahrhunderts, München, C.H. Beck, 2009.
} 
En rédigeant sa critique, Richard J. Evans pensait manifestement au lecteur d'aujourd'hui, guère familiarisé avec les territoires et les frontières des pays du XIX ${ }^{\mathrm{e}}$ siècle, ou avec l'étendue de la Confédération germanique à cette époque. Cependant, Evans oubliait que cela valait tout aussi bien pour les hommes du XIX siècle, qui n'avaient pas nécessairement appris à lire ou à analyser les cartes. En effet, l'émergence des États-nations fut accompagnée, soutenue et illustrée par une abondante production cartographique qui, à partir de la fin du XVIII e siècle, cessa de se limiter aux territoires et aux frontières pour porter de plus en plus fréquemment sur l'économie, la société et la culture. Si les historiens allemands font une exception pour les statistiques, qui constituent une forme d'illustration spécifique, et dont les données font l'objet de représentations spatiales depuis le XIX siècle, ils restent réservés vis-à-vis de la "carte» comme source scientifique. Cela vaut tout particulièrement pour les spécialistes des $\mathrm{XIX}^{\mathrm{e}}$ et $\mathrm{XX}^{\mathrm{e}}$ siècles, tandis que les médiévistes et les modernistes utilisent ce matériel de façon bien plus intensive ${ }^{7}$.

\section{LES HISTORIENS ALLEMANDS ET LES CARTES}

Un certain nombre de travaux récents, par exemple ceux de Peter Haslinger $^{8}$ ou Ulrike Jureit ${ }^{9}$ pour le XX $X^{\mathrm{e}}$ siècle, et ceux de Bernhard Struck ${ }^{10}$ ou Sylvia Schraut ${ }^{11}$ pour le XIX ${ }^{\mathrm{e}}$, ont pourtant bien mis en évidence le potentiel de ces sources cartographiques, dont l'intérêt va bien au-delà de la simple représentation des espaces politiques. En effet, les cartes permettent d'agencer et de structurer les espaces à partir d'informations collectées, analysées et

7. Cf. par exemple Ingrid Baumgärtner et Stefan Schröder, «Weltbild, Kartographie und geographische Kenntnisse", in Johannes Fried et Ernst-Dieter Hehl [dir.], Weltdeutungen und Weltreligionen 600 bis 1500, WBG Weltgeschichte. Eine globale Geschichte von den Anfängen bis ins 21. Jahrhundert, vol. 3, Darmstadt, Wissenschaftliche Buchgesellschaft, 2010, p. 57-83; Ingrid Baumgärtner et Martina Stercken [dir.], Herrschaft verorten. Politische Kartographie im Mittelalter und in der frühen Neuzeit, Zürich, Chronos, 2012; Jörg Dünne, Die kartographische Imagination. Erinnern, Erzählen und Fingieren in der Frühen Neuzeit, Paderborn, Wilhelm Fink, 2011; Tanja Michalsky, Projektion und Imagination. Die niederländische Landschaft der Frühen Neuzeit im Diskurs von Geographie und Malerei, Paderborn, Wilhelm Fink, 2011; Martina Stercken, «Repräsentieren mit Karten als mediales Modell», in Christian Kiening et Martina Stercken [dir.], Modelle des Medialen im Mittelalter, Berlin, Akademie-Verlag, 2010, p. 96-113.

8. Peter Haslinger, Nation und Territorium im tschechischen politischen Diskurs 1880-1938, München, Oldenbourg, 2010; Christian Lotz, Die anspruchsvollen Karten. Polnische, ost- und westdeutsche Auslandsrepräsentationen und der Streit um die Oder-Neiße-Grenze (1945-1972), Magdeburg, Meine, 2011. 9. Ulrike Jureit, Das Ordnen von Räumen. Territorium und Lebensraum im 19. und 20. Jahrhundert, Hamburg, Hamburger Edition, 2012.

10. Bernhard Struck, «Farben, Sprachen, Territorien. Die deutsch-polnische Grenzregion auf Karten des 19. Jahrhunderts", in Christof Dipper et Ute Schneider [dir.], Kartenwelten. Der Raum und seine Repräsentation in der Neuzeit, Darmstadt, Primus, 2006, p. 177-192. Voir aussi l'étude de sa consœur française Morgane Labbe, "Les Frontières de la nation allemande dans l'espace de la carte, du tableau statistique et de la narration", in Catherine Maurer [dir.], Les espaces de l'Allemagne au XIX ${ }^{e}$ siècle. Frontières, centres et question nationale, 2010, Strasbourg, Presse universitaire de Strasbourg, p. 49-72.

11. Sylvia Schraut, Kartierte Nationalgeschichte. Geschichtsatlanten im internationalen Vergleich 18601960, Frankfurt a. M., Campus, 2011. 
hiérarchisées. Elles règlent ainsi les problèmes liés à la contingence, et offrent un instrument d'action future dans la mesure où elles permettent de procéder à toutes sortes de planifications. D'autre part, elles favorisent les comparaisons nationales et internationales car, contrairement aux textes et aux séries statistiques, elles situent les informations dans leur contexte spatial, et les présentent de manière claire.

Dans le cadre d'une réflexion sur la périodisation des XIX et $\mathrm{XX}^{\mathrm{e}}$ siècles, Charles S. Maier a proposé, il y a déjà plus de dix ans, d'écrire l'histoire de l'époque s'étendant des années 1860 aux années 1960 sous l'angle de la territorialité. En s'appuyant sur les travaux de Robert David Sack, il définit la territorialité en ces termes : "Une stratégie géographique puissante visant à contrôler les peuples et les choses en contrôlant l'espace "12. Selon lui, on assiste depuis le milieu du XIX ${ }^{e}$ siècle à une appropriation scientifique et politique croissante des territoires, alors que les deux siècles précédents avaient été marqués par le tracé de frontières. Il s'agissait d'étudier l'intérieur d'un territoire, ses ressources et son potentiel militaire ou énergétique, et de conférer à ces éléments une visibilité afin de négocier et structurer l'ordre social. À cela s'ajoutaient des hiérarchisations spatiales (pôles) et des réseaux de technologies transcendant l'espace. Dans ses réflexions, Maier s'appuie sur les analyses de James C. Scott pour qui la carte constitue, pour les États, un instrument d'organisation et de planification visant à une "catégorisation schématique» de la réalité, au même titre que ces autres «simplifications étatiques" que sont les cadastres et les statistiques ${ }^{13}$. Bien que le terme de "simplification" ne soit guère adapté aux représentations cartographiques, pas plus que l'idée répandue d'une réduction - car, derrière l'activité cartographique se cachent des processus et décisions extrêmement complexes et encore méconnus -, ce sont précisément les cartes et, sous une autre forme, les statistiques qui permettent d'effectuer des comparaisons nationales et internationales. ${ }^{14}$ Cette possibilité a été largement exploitée au XIX ${ }^{\mathrm{e}}$ siècle, comme le montrent par exemple la réception de certaines cartes réalisées par

12. "À powerful geographic strategy to control people and things by controlling area." Sack cité d'après Charles S. Maier, «Transformations of Territoriality. 1600-2000", in Gunilla Budde, Sebastian Conrad et Oliver Janz [dir.], Transnationale Geschichte. Themen, Tendenzen und Theorien, Göttingen, Vandenhoeck \& Ruprecht, 2006, p. 32-55, ici p. 34; Charles S. Maier, «Consigning the Twentieth Century to History: Alternative Narratives for the Modern Era ", The American Historical Review, tome 105 (2000), p. 807-831. Références explicites à la cartographie dans Charles S. Maier, «Leviathan 2.0. Die Erfindung moderner Staatlichkeit ", in Emily S. Rosenberg [dir.], Geschichte der Welt. Weltmärkte und Weltkriege, München, C.H. Beck, 2012, p. 33-286, ici p. 173-175.

13. James C. Scott, Seeing Like a State. How Certain Schemes to Improve the Human Condition Have Failed, New Haven (Conn.), Yale University Press, 1998, p. 77 et 87.

14. Bettina Heintz, «Zahlen, Wissen, Objektivität: Wissenschaftssoziologische Perspektiven », in Andrea Mennicken et Hendrick Vollmer [dir.], Zablenwerk. Kalkulation, Organisation und Gesellschaft, Wiesbaden, Verlag für Sozialwissenschaft, 2007, p. 65-85; Bettina Heintz et Tobias Werron, «Wie ist Globalisierung möglich? Zur Entstehung globaler Vergleichshorizonte am Beispiel von Wissenschaft und Sport ", Kölner Zeitschrift für Soziologie und Sozialpsychologie, tome 63 (2011), p. 359-394. 
Charles Dupin, ou les études sur les techniques de comptage et de mesure dans le cadre de la naissance des Empires ${ }^{15}$.

Malgré l'importance croissante des cartes comme instruments d'action politique, sociale et économique dans le cadre du développement de la territorialité, nous savons étonnamment peu de chose des discussions et processus à l'origine de cette "catégorisation", et de leur évolution (y compris sous forme de représentation graphique), ou encore des interactions entre cartes et États, pour en revenir à l'exemple de Scott. Pourtant - et c'est là notre thèse, qui s'inscrit dans le sillage de Maier -, la territorialité ne saurait se concevoir comme instrument d'organisation et de collecte de données sans représentation graphique, ce qui apparaît clairement dans son argumentation (et la nôtre). Par ailleurs, l'analyse des cartes et des conditions de leur élaboration nous renseigne sur les processus de production et de transfert des savoirs, ainsi que sur l'appropriation progressive et l'étude scientifique des territoires et de leurs ressources, y compris humaines.

Dans ce texte, nous souhaitons montrer l'intérêt d'une approche historique de la cartographie dans sa dimension éminemment politique, en analysant un événement bien connu de l'histoire allemande et européenne : le Congrès de Berlin en $1878^{16}$. Le déroulement et les résultats du Congrès des Nations de Berlin ont fait l'objet de nombreux travaux, en raison de leurs répercussions sur le système des alliances européennes, et d'un vaste recueil de sources publié en $1978^{17}$. Cependant, l'abondant matériel cartographique produit et utilisé depuis le début des négociations, du traité préliminaire de San Stefano à la conclusion définitive des travaux des commissions de délimitation, ne figure pas dans les nouvelles éditions des actes du congrès, même partiellement, et on ne le trouve que dans les dossiers et les fonds d'archives de l'époque ${ }^{18}$. À l'inverse, le matériel cartographique revêtait autrefois une

15. James R. Akerman [dir.], The Imperial Map. Cartography and the Mastery of Empire, Chicago, University of Chicago Press, 2009; Jörn Leonhard et Ulrike von Hirschhausen [dir.], Comparing Empires. Encounters and Transfers in the Long Nineteenth Century, Göttingen, Vandenhoeck \& Ruprecht, 2011; Ute Schneider, «Die Farbe der Religion. Topografie und Topik der 'Deux France'», Journal of Modern European History, tome 9, $\mathrm{n}^{\circ} 1$ (2011), p. 117-139.

16. Cet article présente des axes de réflexion développés dans une publication dont la version allemande paraitra au cours de l'année 2014.

17. Liste non exhaustive: Karl Otmar von Aretin [dir.], Bismarcks Aussenpolitik und der Berliner Kongress, Wiesbaden, Steiner, 1978; William Leonard Langer, European Alliances and Alignments 1871-1890, New York, Knopf, 1931; Walter Markov, Grundzüge der Balkandiplomatie. Ein Beitrag zur Geschichte der Abhängigkeitsverhältnisse, Leipzig, Leipziger Universitäts-Verlag, 1999; Ralph Melville et HansJürgen Schröder [dir.], Der Berliner Kongress von 1878. Die Politik der Grossmächte und die Probleme der Modernisierung in Südosteuropa in der zweiten Hälfte des 19. Jahrhunderts, Wiesbaden, Steiner, 1982; Wolfgang J. Mommsen, Grossmachtstellung und Weltpolitik. Die Aussenpolitik des Deutschen Reiches 1870 bis 1914, Frankfurt a. M., Ullstein, 1993; Alexander Novotny, Quellen und Studien zur Geschichte des Berliner Kongresses 1878, Graz-Köln, Böhlau, 1957; Imanuel Geiss [dir.], Der Berliner Kongress 1878. Protokolle und Materialien, Boppard, Boldt, 1978.

18. Actenstücke in orientalischen Angelegenheiten. Präliminarfriede von St. Stefano. Congres-Protokolle und Vertrag von Berlin, Wien, 1878; Edward Hertslet [dir.], The Map of Europe by Treaty. Providing the Various Political and Territorial Changes Which Have Taken Place Since the General Peace of 1814, tome 4 (1875-1891), London, 1891; Ministère des Affaires étrangères [dir.], Affaires d'Orient. Congrès de Berlin, Paris, 1878. Ces travaux n'ont pas été publiés par l'Allemagne, on dispose uniquement d'une édition 
grande valeur informative; aussi la question de cette documentation fut-elle à l'ordre du jour en amont des négociations, puis pendant toute la durée du congrès.

\section{Le Congrès de Berlin (I878)}

\section{Utilisation et production des cartes pendant le congrès}

Depuis 1875 , la région dite des Balkans, considérée comme orientale, avait connu plusieurs révoltes que les puissances européennes entendaient pacifier. Après l'échec d'une conférence commune organisée à Constantinople en 1876, la guerre éclata au printemps 1877 . Elle opposait la Russie et l'Empire ottoman. Alors que celui-ci avait réaffirmé sa domination sur les Balkans avec sa nouvelle Constitution, la Russie soutenait les mouvements indépendantistes panslavistes ${ }^{19}$. Étant donné la supériorité militaire des Russes, la guerre prit fin avec le cessez-le-feu de janvier 1878, inscrit dans le traité de paix de San Stefano au début du mois de mars, comme l'indépendance de la Serbie et de la Roumanie, et la création d'une vaste principauté de Bulgarie. Les puissances européennes, inquiètes, avaient suivi de près la guerre, et, dès le cessez-le-feu, elles s'efforcèrent de glaner des informations sur les conditions de paix imposées par les Russes. En apprenant ce qu'il en était, la Grande-Bretagne et surtout l'Autriche exigèrent une conférence européenne sur les modifications et créations territoriales prévues, car elles considéraient que ces projets, contraires au traité de Paris de 1856 et au protocole de Londres de 1871, portaient atteinte aux intérêts européens.

À la fin mars, les Russes finirent par faire parvenir au comte de Derby, ministre britannique des Affaires étrangères, et aux représentants des autres puissances européennes le traité préliminaire de paix, accompagné d'une carte explicative. Le traité lui-même était assorti de plusieurs cartes commentées dans les divers articles du texte ${ }^{20}$. La majorité de ces schémas cartographiques isolés, qui ne représentaient que les frontières nationales, les

des négociations du congrès avec une carte fournissant un aperçu général : Johannes Lepsius, Albrecht Mendelssohn Bartholdy et Friedrich Thimme [dir.], Der Berliner Kongreß und seine Vorgeschichte, Die große Politik der europäischen Kabinette 1871-1914 (Sammlung der diplomatischen Akten des Auswärtigen Amtes) 1, tome 2, Berlin, Deutsche Verlagsgesellschaft für Politik und Geschichte, 1922. Sur la Russie, cf. Charles Jelavich et Barbara Jelavich [dir.], Russia in the East, 1876-1880. The Russo-Turkish War and the Kuldja Crisis As Seen Through the Letters of A.G. Jomini to N.K. Giers, Leiden, Brill, 1959. 19. Cf. Winfried Baumgart, Europäisches Konzert und nationale Bewegung. Internationale Beziehungen 1830-1878, Handbuch der Geschichte der Internationalen Beziehungen, tome 6, Paderborn/München/ Wien/Zürich, Schöningh, 2007, p. 420-423.

20. «Preliminary Treaty of Peace between Russia and Turkey», in House of Commons, Turkey. Parliamentary Papers Online, $n^{\circ} 22,1878$. Cette documentation comprend les cartes annexées au traité. En outre, des cartes sur lesquelles la Russie et l'Autriche avaient inscrit et communiqué dès 1877 leurs prétentions territoriales sur les États en formation dans les Balkans jetèrent le trouble dans les discussions préliminaires. Ces antécédents ne peuvent être développés ici. À ce sujet, $c f$. Johannes Lepsius, Albrecht Mendelssohn Bartholdy et Friedrich Thimme [dir.], Der Berliner Kongreß..., op. cit., p. 253, 257 et 260. 
fleuves et quelques villes, se fondaient sur la "Carte générale de la Turquie européenne et du Royaume de Grèce», dont le géographe berlinois Heinrich Kiepert (1818-1899) avait publié une édition révisée en 187021. Cette carte, et la "Völker- und Sprachen-Karte von Österreich und den UnterDonau-Ländern (Carte des peuples et langues d'Autriche et des pays du sud du Danube)", publiée en 1869, figuraient parmi les rares sources d'information disponibles sur la population des Balkans, comme le fit remarquer Bismarck lors d'un débat au Reichstag sur la question de l'Orient, en février 1878 : «Les informations ethnographiques dont nous disposons sur le sujet ne sont pas authentiques, elles sont lacunaires; les meilleures, du moins à notre connaissance, sont les cartes de Kiepert, qui sont l'œuvre de mains allemandes $»^{22}$. Bien que la carte de Kiepert couvrît aussi la région située à l'est de la mer Noire, les frontières fixées par le traité de San Stefano entre la Russie et l'Empire ottoman autour de Batoumi - port de la mer Noire qui devait passer sous domination russe - furent définies à partir d'une carte d'état-major russe (IIl. 1). Avec le changement de référence au profit de cette dernière, les modifications affectant les territoires et les rapports de domination furent bien documentées et représentées. À l'inverse, le texte du traité privilégiait une langue plus circonspecte; l'article correspondant prévoyait en effet que «les limites définitives du territoire annexé à la Russie, et marqué sur la carte ci-jointe, soient fixées par une commission composée de délégués russes et ottomans $»^{23}$.

Après l'annonce de l'accord préliminaire de paix, les échanges diplomatiques entre les différents représentants des puissances européennes, déjà abondants, s'intensifièrent et se cristallisèrent sur la question de savoir s'il fallait organiser une conférence ou un congrès, et, si oui, en quel lieu. En amont, le gouvernement britannique avait arraché à la Russie la promesse que chaque article du traité préliminaire serait négociable lors de ce congrès ${ }^{24}$. Finalement, les puissances européennes se réunirent à Berlin pour un mois, en juin 1878. Sous la houlette de Bismarck, qui se définissait lui-même comme un "honnête agent d'affaires", les articles du traité de San Stefano furent examinés non pas par ordre chronologique, mais par ordre d'importance. C'est ainsi que la question bulgare fut abordée en premier lieu ${ }^{25}$.

21. Heinrich Kiepert, General-Karte Von Der Europäischen Türkei, Berlin, 1870. Une version numérique des éditions de 1853 et de 1855 est consultable sur http://www.lib.uchicago.edu/e/su/maps/kiepert/ (dernière consultation le 29/08/2012). Sur Kiepert, voir aussi Lothar Zögner [dir.], Antike Welten, neue Regionen. Heinrich Kiepert 1818-1899, Berlin, 1999. L'importance des cartes réalisées par Kiepert pour cette région a notamment été soulignée par Herbert Wilhelmy dans "Das Kartenwesen Bulgariens", Mitteilungen des Reichsamts für Landesaufnahme, tome 3, 1938, p. 139-159.

22. "Aus der Sitzung des Reichstags vom 19. Februar 1878», Das Staatsarchiv (Die Orientalische Frage. Protokolle und Reden.), tome 34, Leipzig, 1878, p. 1-55, citation p. 7.

23. Cité d'après la traduction anglaise du traité dans «Preliminary Treaty of Peace... ", loc. cit., p. 15.

24. Salisbury à "Her Majesty's Embassies", le $1^{\text {er }}$ avril 1878, in Edward Hertslet [dir.], British and Foreign State Papers, vol. 69, p. 807-814.

25. "Protokoll Nr. 1, Sitzung vom 13. Juni 1878», in Imanuel Geiss [dir.], Der Berliner Kongress $1878 \ldots$, op. cit., p. 177. 


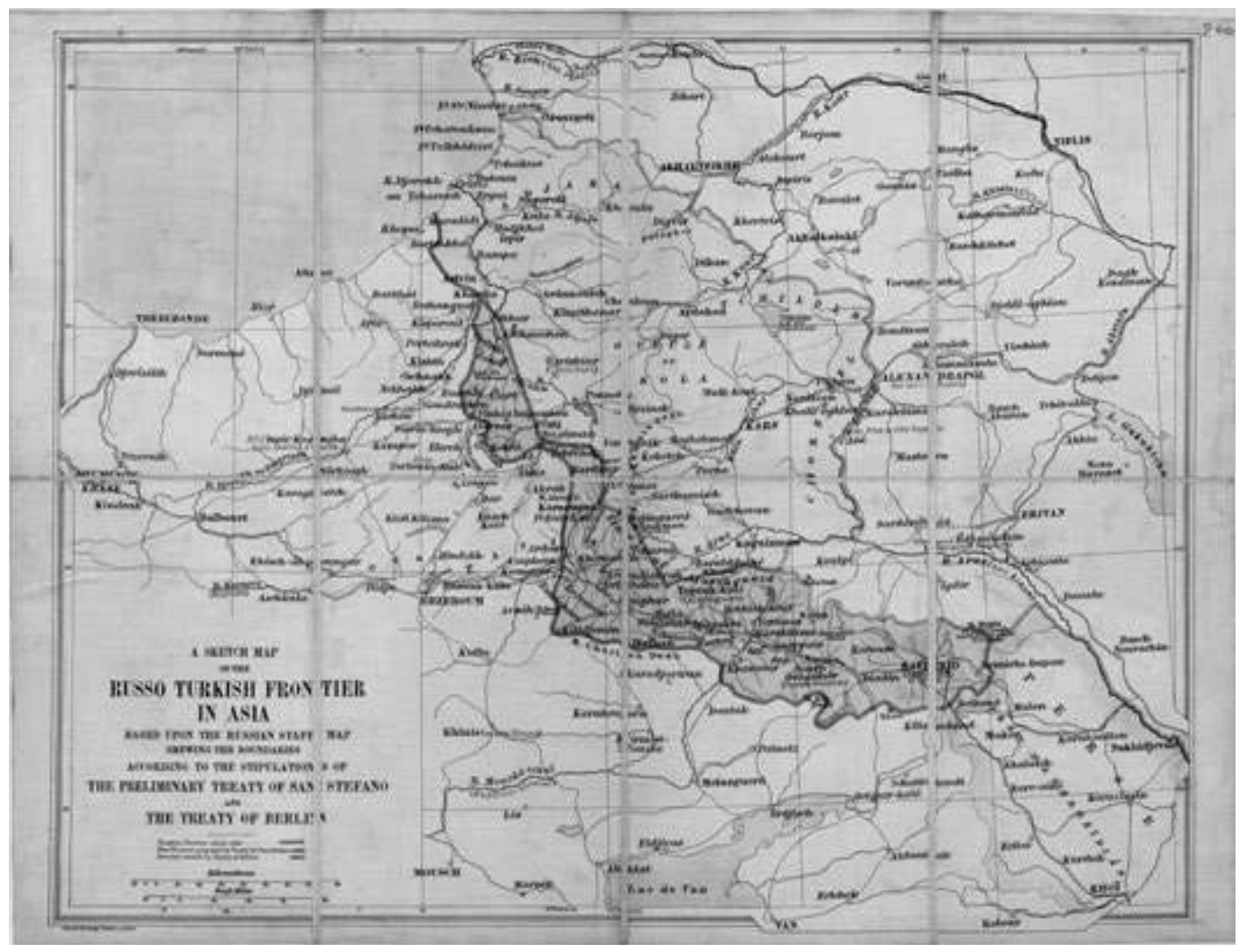

Ill. 1 : «A sketch map of the Russo-Turkish frontier in Asia, based upon the Russian Staff map showing the boundaries according to the stipulations of the preliminary Treaty of San Stefano and the Treaty of Berlin. 16 miles to one inch. E. Stanford, London», National Archives, London.

Les séances initiales portèrent sur des questions de base telles que l'organisation de l'État et le traitement des minorités religieuses. Puis, à la fin du mois de juin, la définition concrète des frontières fut inscrite à l'ordre du jour. Bismarck avait soigneusement planifié le congrès et son organisation. La disposition des sièges avait été pensée jusque dans ses moindres détails, et "un grand tableau avec des cartes» avait été placé "dans la salle, en face de la table du congrès ${ }^{26}$. Cependant, il fallut rapidement se rendre à l'évidence : «Il était impossible de décrire précisément les frontières en assemblée plénière». On créa alors une commission comprenant un délégué de chaque pays pour "élaborer le tracé des frontières et le présenter au congrès " ${ }^{27}$. Par la suite, toutes les questions litigieuses sur lesquelles l'assemblée plénière

26. Hajo Holborn [dir.], Aufzeichnungen und Erinnerungen aus dem Leben des Botschafters Joseph Maria von Radowitz, Stuttgart, Deutsche Verlags-Anstalt, 1925, p. 38.

27. "Protokoll der 8. Sitzung am 28. Juni 1878 », in Imanuel Geiss [dir.], Der Berliner Kongress 1878..., op. cit., p. 254. La commission était composée comme suit : «Pour l'Allemagne, le prince de Hohenlohe - pour l'Autriche, le baron de Haymerle - pour la France, le comte de Saint-Vallier - pour la Grande-Bretagne, Lord Odo Russell - pour l'Italie, le comte de Launay - pour la Russie, le comte 
perdait beaucoup de temps furent «imposées» à cette commission, dont les séances pouvaient être "très houleuses $»^{28}$. Toutefois, en l'absence d'accord systématique sur le tracé des frontières, ces cas étaient renvoyés vers l'assemblée plénière; aussi cette dernière adopta-t-elle, au début juillet, un nouveau mode de suffrage permettant à la commission de prendre des décisions à la majoritée 29 . Malgré cette simplification de la procédure, la commission ne put venir à bout de son énorme volume de travail qu'en «se réunissant quotidiennement et souvent jusqu'au milieu de la nuit» durant les deux dernières semaines du congrès ${ }^{30}$. Elle présentait régulièrement les résultats de son travail à l'assemblée plénière et, en général, ses propositions étaient intégrées au traité sous la forme proposée. Au-delà des discussions de fond et des formes de communication mondaine, «les commissions spécialisées sur le tracé des frontières travaillaient d'arrache-pied [...] à l'accord", se rappelait bien des années plus tard le diplomate allemand Joseph Maria von Radowitz ${ }^{31}$. S'il était difficile de s'entendre au sein de la commission, c'était non seulement du fait d'idées et d'attentes divergentes sur les territoires, mais surtout à cause des écarts existant en matière de savoir régional, du niveau de connaissances parfois médiocre, et des sources d'information lacunaires. Sur ce dernier point, en tout cas, les organisateurs allemands avaient fait le maximum. Le nécessaire d'écriture fourni à chaque délégué à sa place contenait, outre les protocoles des négociations de 1876, «une copie du traité de San Stefano avec une carte, une copie du traité de Paris de 1856, et un exemplaire de la carte de la péninsule des Balkans réalisée par Kiepert " "22. Ces cartes, les délégués les utilisèrent beaucoup pendant les négociations, pour expliciter leur propos sur le tracé des frontières, notamment les spécificités topographiques et les exigences stratégiques. Ils se servirent également de la carte d'état-major autrichienne, en cours d'élaboration depuis 1870 sur la base d'un vaste ensemble de mesures territoriales, mais encore incomplète au moment du congrès ${ }^{33}$. En l'absence d'autres cartes fiables ou mises à jour, les

Schouvaloff - pour la Turquie, Mehmed Ali Pascha.» Source : «Protokoll der 9. Sitzung am 29. Juni 1878 ", in Imanuel Geiss [dir.], Der Berliner Kongress 1878..., op. cit., p. 259.

28. Le prince de Hohenlohe, qui dirigeait la commission, ne parle guère des travaux de cette dernière dans son journal, du moins dans les parties qui ont été publiées. En revanche, il évoque la multitude de problèmes et de conflits qui déchirèrent la commission au début juillet, et qui motivèrent peut-être la décision de l'assemblée plénière. Friedrich Curtius [dir.], Denkwürdigkeiten des Fürsten Chlodwig zu Hohenlohe-Schillingsfürst, Stuttgart, Deutsche Verlags-Anstalt, 1906, p. 244. On peut lire un certain nombre de commentaires sur les travaux de la commission dans L. Raschdau, «Die Botschafterkonferenz in Konstantinopel und der russisch-türkische Krieg (1877-1878). Aus dem literarischen Nachlaß des Unterstaatssekretärs Dr Busch ", Deutsche Rundschau, n 141, 1909, p. 12-28, 207-222, 361-379, ici p. 376.

29. «Protokoll der 14. Sitzung am 6. Juli 1878», in Imanuel Geiss [dir.], Der Berliner Kongress 1878..., op. cit., p. 318 ; «Protokoll der 16. Sitzung am 8. Juli $1878 »$, idem, p. 334.

30. Hajo Holborn [dir.], Aufzeichnungen und Erinnerungen..., op. cit., p. 63.

31. Idem, S. 54. Sur les discussions de fond, $c f$. «Protokoll der 14. Sitzung am 6. Juli 1878 ", in Imanuel Geiss [dir.], Der Berliner Kongress 1878..., op. cit., p. 314-315.

32. Hajo Holborn [dir.], Aufzeichnungen und Erinnerungen..., op. cit., p. 38-39.

33. Herbert Wilhelmy, «Das Kartenwesen...", loc. cit., p. 141-147. 
participants s'accordèrent pour utiliser ce document comme base des négociations. Non seulement il leur permit de visualiser les choses, mais il les aida à définir les frontières par des mots dans le traité. Dans l'ensemble, le texte prévoyait un tracé suivant les frontières naturelles, mais, parfois, il dut se référer à des points trigonométriques figurant sur la carte, par exemple dans le cas du Monténégro, où il était impossible de se fonder sur des caractéristiques topographiques ${ }^{34}$.

Durant les tractations sur la ville de Batoumi, la Russie utilisa son propre matériel cartographique, fondé sur la carte d'état-major russe (Ill. 1) ${ }^{35}$. À Berlin, on s'accorda pour négocier à partir de ce document pour la définition de la frontière russo-turque. Néanmoins, plus tard, les travaux de délimitation devaient révéler que les topographes russes étaient encore loin d'avoir achevé leur ouvrage ${ }^{36}$. Avec ce document cartographique, la diplomatie russe prit les devants, non seulement sur le plan de l'expansion territoriale, mais aussi sur celui de l'étude et de la représentation cartographiques de l'ensemble du territoire national. Toutefois, le tracé des frontières en Asie - généralisation désignant la frontière russo-turque - se révéla difficilement réalisable à partir du matériel cartographique disponible, d'autant plus que les prétentions de la Russie se heurtaient à la résistance de la Grande-Bretagne. Finalement, la commission de délimitation dut se rendre à l'évidence : elle ne disposait «ni des connaissances du terrain, ni des cartes et documents nécessaires» pour mener à bien son travail. Aussi, le marquis de Salisbury et le comte Schouvaloff décidèrent d'un commun accord qu'une commission militaire procéderait sur place à la définition et au tracé des frontières pour régler les litiges. Cet accord fut annexé au traité de Berlin ${ }^{37}$. Le matin de la conclusion du traité, il s'avéra néanmoins que ce compromis portait uniquement sur la description des frontières dans le texte du traité, et sur la délégation des décisions finales à la commission de démarcation présente sur place. Le scandale éclata juste avant la dernière séance du congrès, quand les officiers se réunirent avec leurs cartes afin d'y inscrire un tracé identique. Alors que le délégué britannique était en train de reporter les frontières sur la carte

34. « und erreicht sodann den Punkt 2166 der österreichischen Generalstabskarte, indem sie der Hauptkette und der Wasserscheidelinie zwischen dem Lim einerseits und dem Drin und der Cievna (Zem) andererseits folgt». "Protokoll der 12. Sitzung am 4. Juli 1878 und Artikel 28 des Berliner Vertrags», in Imanuel Geiss [dir.], Der Berliner Kongress 1878..., op. cit., p. 293 et 390.

35. Voir la représentation cartographique de ce tracé dans Edward Hertslet [dir.], The Map of Europe by Treaty..., op. cit., p. 2794.

36. Cela explique aussi le peu d'informations fournies par cette carte, qui précise uniquement certaines caractéristiques topographiques. "Protokoll der 14. Sitzung am 6. Juli 1878», in Imanuel Geiss [dir.], Der Berliner Kongress 1878..., op. cit., p. 314; "Annex to Article XIX of the Treaty of San Stefano", in House of Commons, Turkey. Parliamentary Papers Online, n 22, 1878, p. 6 et suivantes; pour approfondir, voir la lettre du colonel Holmes à Salisbury en date du 10 octobre 1878, dans "Correspondence respecting the European Commissions appointed for the demarcation of frontiers under the Treaty of Berlin ", in House of Commons, Turkey. Parliamentary Papers Online, nº 2, 1879, p. 29.

37. «Protokoll der 18. Sitzung am 11. Juli 1878 ", in Imanuel Geiss [dir.], Der Berliner Kongress 1878..., op. cit., p. 355-356; Edward Hertslet [dir.], The Map of Europe by Treaty..., op. cit., p. 2758. 
d'état-major autrichienne conformément aux termes du traité, le délégué russe apporta une carte déjà complétée, dont les tracés ne correspondaient pas toujours à ceux des autres : «Le colonel Bogulobow partit sans demander à ce que ses cartes soient vérifiées. ${ }^{38}$ Pendant que le représentant ottoman s'élançait à sa suite, les autres délégués relevèrent les différences présentées par sa carte. Elles portaient sur des indications de distance, mais aussi sur des lignes de partage des eaux qui, d'après les délégués encore présents, constituaient un problème mineur.

Les délégués se heurtèrent à d'autres obstacles avec la frontière sud-ouest de la Roumélie, qui dut être tracée sur la carte de Kiepert, parce que la carte d'état-major autrichienne portant sur cette région n'était pas encore publiée, et que seuls les Autrichiens et les Britanniques possédaient une copie de la version provisoire. Des différences topographiques entre ces deux cartes entraînèrent une "anomalie» que les délégués encore présents parvinrent toutefois à résoudre par le dialogue.

Les divergences entre les frontières définies sous forme cartographique et sous forme textuelle constituèrent un défi de taille durant les travaux de délimitation, ainsi que les écarts constatés entre les cartes des différents délégués.

\section{La production cartographique des commissions de délimitation}

La plupart des commissions chargées de définir les frontières entre les différents États prirent leurs fonctions dès la fin du congrès. Dans certains cas, leur composition avait déjà été fixée à Berlin. Elle prévoyait non seulement la participation de délégués des États frontaliers, mais aussi celle d'au moins un représentant d'un pays signataire neutre. Généralement, on envoya sur place des officiers qui avaient été associés aux négociations à Berlin, et qui disposaient de connaissances topographiques, ainsi que d'une certaine expérience en matière de procédés d'arpentage. Avant même de se mettre en route, ils reçurent des directives claires sur l'exécution de leur tâche, et furent informés des intérêts de leurs gouvernements respectifs, comme le montre l'ordre de mission adressé par Salisbury au représentant britannique, le Colonel Home :

"Je dois vous demander de vous montrer, lorsque vous aborderiez avec vos collègues les questions de cette nature, d'une disposition conciliante. Le gouvernement de Sa Majesté n’a aucun désir de débattre de vétilles ; sa volonté est de voir cette affaire résolue de manière à ce que ne se présentent pas à l'avenir des questions insignifiantes, lesquelles pourraient donner lieu à des complications, ou pourraient envenimer des situations déjà conflictuelles. Il a été suggéré par le gouvernement français que la clôture des débats sur chaque question adviendrait par décision de la majorité des membres de la Commission; le gouvernement russe a toutefois fait part de son opinion que

38. Memorandum J.C. Ardagh vom 13.7.1878, Inclosure 2 in $n^{\circ} 21$, in Turkey n 2 (1879). Correspondence respecting the European Commissions appointed for the demarcation of frontiers under the Treaty of Berlin, S. 12. 
dans les cas où la Commission n'est pas unanime, il faudrait se référer aux représentants des pouvoirs signataires à Constantinople.

Je ne puis que vous enjoindre de faire tout votre possible pour éviter la perte de temps que constituent de tels appels aux représentants, en proposant ou en ratifiant tout compromis qui vous paraitrait équitable et efficace. $\aleph^{39}$

Avec le recul, ce furent précisément les conflits et discussions à éviter qui ralentirent les travaux de délimitation, si l'on fait abstraction des problèmes quotidiens tels que les aléas météorologiques, les conditions hivernales, les longues périodes d'enneigement ou les maladies.

L'un des principaux défis qui se posèrent à l'ensemble des commissions consista à gérer la concordance entre le texte du traité, les cartes et la situation sur le terrain. Dès la première séance, le débat sur le rapport entre texte et carte fut à l'ordre du jour. Si l'approche adoptée et les différentes étapes du processus de délimitation furent négociées au début des travaux, le caractère contraignant du texte fit régulièrement l'objet de discussions. Deux positions s'affrontèrent; la plupart des membres rallièrent le camp du délégué italien Orero qui, dans sa commission, choisit de qualifier le traité de «texte sacré». Dès lors, on considéra que la carte d'état-major autrichienne sur laquelle figuraient les frontières était un "document complémentaire du Traité du Berlin", et qu'elle devait «être prise en considération dans tous les cas où il s'agi[ssai]t [d']expliquer le texte et l'esprit du Traitée ${ }^{40}$." Quant aux membres de la commission représentant leur propre État, ou poursuivant des intérêts précis au sujet d'une frontière ou d'une région en particulier, ils adoptèrent une autre stratégie. Sous prétexte d'aborder les difficultés prévisibles liées au tracé, ils relançaient régulièrement le débat sur l'interprétation du texte, le marquage figurant sur la carte et les délimitations à concrétiser sur place.

La hiérarchisation entre le texte du traité, la carte et la réalité du terrain souleva également des débats sur la manière de procéder, et l'ordre dans lequel exécuter les travaux. Dans chaque commission, certains préconisaient d'aller directement sur le terrain. Cependant, étant donné le caractère prioritaire du texte, ils durent se plier à une étude intensive du traité et des cartes en amont pour préparer le tracé proprement dit. On définit une procédure en plusieurs étapes : premièrement, étudier la frontière sur des cartes topographiques et résoudre toutes les questions litigieuses. Deuxièmement, se rendre sur place pour arpenter la future frontière. Troisièmement, inscrire sur la carte son tracé définitif, en concertation. Quatrièmement, concrétiser la frontière par un marquage visible sur le terrain ${ }^{41}$.

39. Salisbury à Home, le 9 septembre $1878, c f$. idem, p. 8 .

40. Protocole $\mathrm{n}^{\circ} 2$, Séance du $1^{\text {er }}$ mai 1879, $c f$. «Further Correspondence respecting the European commissions appointed for the demarcation of frontiers under the Treaty of Berlin ", in House of Commons, Turkey. Parliamentary Papers Online, n 2, 1880, p. 61; Protocole n 15, Séance du 21 avril 1879, idem, p. 34.

41. Protocole $n^{\circ} 14$, Séance du 8. avril 1879, idem, p. 79; Protocole n 31, Séance du 18 mai 1879, idem, p. 204. 
Pour effectuer une analyse cartographique détaillée, encore fallait-il disposer du matériel nécessaire, et ce à une échelle permettant de régler les questions frontalières. Au début des travaux, les commissions n'avaient guère d'autre carte que celle utilisée à Berlin, aussi travaillèrent-elles essentiellement à partir de la carte d'état-major provisoire fournie par l'Autriche. Or, le tracé y figurant n'ayant pas fait l'unanimité lors de la dernière séance de la commission de démarcation à Berlin, il n'avait pas été ratifié comme partie intégrante du traité.

Les questions en suspens depuis la conférence firent plus particulièrement l'objet de nouveaux débats au sein de la commission de démarcation monténégrine, au sujet de la frontière avec la Turquie. Le délégué turc, Carathéodory Pacha, fit valoir l'absence de ratification dans une longue lettre adressée à l'ambassadeur turc en Grande-Bretagne, dans laquelle il recensa un nombre important de contradictions entre le texte et la carte, ainsi que des erreurs sur cette dernière. Seule la réalisation de nouveaux relevés de terrain permettrait de "préparer une solution juste et pratique », conclut-il ${ }^{12}$. Même si l'ambassadeur parvint manifestement à convaincre le ministre britannique des Affaires étrangères de cette nécessité, Salisbury jugea que cette exigence était en nette contradiction avec le comportement du gouvernement turc, "qui, si je ne m’abuse, avait refusé, et continu[ait] de refuser à la commission et à ses délégués l'accès à certains des principaux points stratégiques du pays en question ${ }^{43}$. Malgré un dialogue intensif entre Londres et Constantinople, le travail de cette commission fut extrêmement conflictuel du début à la fin, et les compromis obtenus furent le résultat d'âpres négociations que nous ne développerons pas ici.

À l'instar de la frontière turque, le tracé de la frontière russe s'avéra complexe, car il devait traverser des territoires qui ne figuraient pas sur la carte autrichienne. Le représentant russe exploita cette lacune pour présenter «sous les yeux» de la commission des photographies de cartes topographiques réalisées par la Russie. Il promit d'en fournir un exemplaire à chaque délégué, à condition qu'on l'adoptât comme référence ${ }^{44}$. La commission accepta. Cependant, Hamley ne prit pas pour argent comptant les données inscrites sur la carte. Pour s'assurer qu'elle constituait une base de travail fiable, il la fit comparer à d'autres sources d'information :

"Au sujet de cette frontière émerge un tel enchevêtrement de questions de principe (qui appellent peut-être une modification du texte même du Traité) et de détails purement locaux, qu'il serait extrêmement difficile d'en arriver à une décision sans connaissance autochtone, même avec l'appui de l'étude la meilleure et la plus complète. En outre, même en supposant un accès aux savoirs locaux, l'affaire pourra difficilement être résolue par correspondance,

42. Carathéodory Pacha à Musurus Pacha le 5 juin 1879, $c f$. idem, p. 107-108.

43. Salisbury à A. H. Layard, le 26 juin 1879 , idem, p. 117.

44. Protocole $\mathrm{n}^{\circ} 14$, Séance du 18. avril 1879, idem, p. 30. 
et sans une forme de conférence avec les représentants des pouvoirs signataires. $\|^{45}$

Il en arriva à la conclusion que les documents avaient été réalisés avec un soin inégal, et qu'ils présentaient encore un certain nombre de «lacunes mineures, qu'on [était] en train de combler». Son homologue russe ne lui paraissant pas très au fait des techniques d'arpentage, Hamley craignait que la mesure des massifs montagneux, effectuée au jugé, ne comportât des "erreurs considérables»; aussi estima-t-il nécessaire de procéder à des corrections, notamment sur les points revêtant une importance stratégique.

Si les cinq topographes affectés par les Britanniques à cette seule commission finirent par être mobilisés, ce fut non seulement du fait de ces corrections, mais aussi à cause des retards permanents des topographes russes. Ces derniers ne cessaient de différer les relevés requis par la commission, les Britanniques les soupçonnaient d'être trop accaparés par d'autres travaux de mesure. Durant l'été 1879 , après un nouveau retard, Hamley perdit patience et chargea les topographes britanniques de cartographier les zones manquantes, c'est-à-dire une multitude de petits points blancs figurant sur les cartes russes. Ce projet se heurta à une vive opposition de la part des délégués russes, mais la commission appuya la décision de Hamley, qui espérait ainsi faire avancer plus vite les travaux russes. La pression induite par cette mise en concurrence porta ses fruits, et seuls quelques rares tronçons de la frontière ne furent pas achevés avant la dissolution de la commission, faute de «carte précise» de la région; en outre, on s'était lassé d'attendre les travaux des topographes russes ${ }^{46}$. Si les opérations de mesure et de délimitation se révélèrent plus longues et fastidieuses que prévu sur le terrain, c'est parce que la commission fut confrontée à une multitude d'aléas nécessitant des données supplémentaires et des informations plus complètes.

À l'issue de ce travail intensif sur les frontières prévues par le traité, et de leur transcription sur les cartes, l'espace ainsi représenté devint une sphère d'expérience pour les membres de la commission. Ils rencontrèrent des problèmes que l'étude des documents ne laissait pas prévoir. En effet, ils avaient beau comparer dans les moindres détails la carte et le texte, dont les dispositions avaient été élaborées à partir du matériel cartographique disponible, cela ne les empêchait pas d'être confrontés à des contradictions, à des erreurs et à des imprécisions une fois sur les lieux. Les délégués avaient des réactions très diverses face à ce savoir issu de l'expérience; en général, ils les recoupaient minutieusement avec les informations fournies par leurs documents. Ils ne modifiaient pas nécessairement leurs relevés de terrain pour y intégrer ces connaissances; et, s'ils le faisaient, c'était au terme d'un processus de négociation et d'arbitrage entre les intérêts des différents pays concernés, en 
tenant compte des informations fournies par les textes et les cartes. En même temps, on accordait aux connaissances du terrain - «local knowledge» en anglais - une importance telle que les modifications s'imposaient plus facilement sur place qu'au stade de l'étude des cartes :

"There arise concerning this frontier such a mixture of questions of principle (involving, perhaps, a modification of the text of the Treaty itself), and of purely local details, that would be exceedingly difficult to come to any decision without local knowledge, even if provided with the best and most complete survey which could be made; moreover, even supposing the local knowledge to be available, the question at issue could hardly be settled by correspondence, without some sort of conference of the Representatives of the Powers $»^{47}$.

\section{Statistique et cartographie}

Pour recueillir des informations de terrain (local knowledge), la commission effectuait entre autres des études statistiques visant à trancher des questions d'appellation, et d'appartenance religieuse ou ethnique. Dans ce cadre, elle recourait à des techniques de classification et de quantification qui faisaient l'objet de discussions enflammées depuis les années 1850 au sein de la communauté scientifique ${ }^{48}$.

En Allemagne comme dans d'autres pays d'Europe, ces débats se déroulaient dans des lieux et des institutions aussi divers que des revues scientifiques, des sociétés de statistique, des instituts ou des administrations. Ces débats s'inscrivaient par ailleurs dans un cadre largement transnational, permis par le grand nombre des rencontres et des contacts personnels. Il existait notamment un événement très peu étudié, mais extrêmement important pour les scientifiques de l'époque : les Congrès internationaux de statistique, organisés entre 1853 et 1876 sous forme de "congrès itinérants » dans plusieurs capitales européennes ${ }^{49}$. Les participants ne se contentaient pas d'échanger des points de vue et des connaissances sur des études ou leurs résultats; ils faisaient des choix méthodologiques ou normatifs, afin de permettre des comparaisons internationales, ou de les faciliter. En effet, la possibilité de se livrer à des comparaisons temporelles, nationales ou internationales faisait partie intégrante de ces méthodes, et de ce qu'on en attendait.

47. Sale à Salisbury, le 2 juillet 1879, idem, p. 137.

48. Ute Schneider, "Inquiries or Statistics? Agricultural Surveys and Methodological Considerations in the Nineteenth Century", in Nadine Vivier [dir.], Inquiries, London, 2013 (en cours d'impression). 49. Adolf Ficker, Georg von Toggenburg-Sargans et Karl von Czoernig, Rechenschafts-Bericht über die dritte Versammlung des internationalen Congresses für Statistik, abgehalten zu Wien vom 31. August bis 5. September 1857, Vienne, 1858, p. 4. 
Pour les organisateurs comme pour les participants, ces congrès devaient permettre l'élaboration et l'adoption de critères de comparaison valides ${ }^{50}$.

Dès le premier congrès, ils soulevèrent des questions sur les représentations graphiques et leur réalisation, en citant abondamment les géographes, souvent présents à ces rencontres. Ainsi, le comité préparatoire du troisième congrès, qui se tint en 1857 à Vienne, mena une réflexion approfondie sur le rapport entre cartographie et statistique, et constata par écrit "que dans de très nombreux cas, il pouvait être opportun de présenter les résultats d'observations scientifiques sous forme cartographique, afin de les rendre plus parlants" ${ }^{51}$. Le comité inscrivit à l'ordre du jour la question de la "caractérisation graphique des cours d'eau ", un problème qui devait donner du fil à retordre aux commissions de délimitation des Balkans, vingt ans plus tard.

La statistique et la cartographie étaient complémentaires en tant que techniques scientifiques d'étude du territoire et de ses ressources, dont elles offraient une représentation concrète. Ce point de vue fut formulé par Johannes Fallati (1809-1855), juriste et professeur de statistique et d'histoire politique à Tübingen, dans son rapport sur le premier Congrès de statistique de Bruxelles, en 1853 : «En réalité, l'objet de la statistique est l'existence des hommes au sein de l'État et de la société, existence qui reste toutefois indissociable de la vie de la nature sur la Terre $»^{52}$.

Cependant, cette interaction fructueuse entre la statistique et la cartographie ne faisait pas uniquement l'objet de congrès scientifiques; en Europe et dans les empires coloniaux, elle était déjà utilisée comme instrument de domination en lien avec la topographie ${ }^{53}$. Les géomètres des commissions de délimitation purent s'appuyer sur ces expériences et ces connaissances. La documentation et l'analyse minutieuses de leurs études fournirent aux commissions des critères et des bases de décision pour le tracé des frontières, qui ne faisaient pas toujours l'unanimité parmi les délégués, car ils contredisaient les idées et classifications traditionnelles ${ }^{54}$.

Aux yeux des membres des commissions, la «local knowledge» (les connaissances de terrain) et l'expérience personnelle jouaient toutefois elles aussi un rôle majeur concernant la "vie de la nature». Comme nous l'évoquions plus haut, les fleuves et leur représentation mettaient l'ensemble des commissions à rude épreuve. Ainsi, la commission serbe constata que la Morava se composait de deux cours d'eau absents de la carte autrichienne,

50. Voir mon article détaillé sur le sujet : Ute Schneider, «Inquiries or Statistics? ... », loc. cit.

51. Sur l'utilisation de la cartographie et des graphes en général en statistique, $c f$. Adolf Ficker, Georg von Toggenburg-Sargans et Karl von Czoernig, Rechenschafts-Bericht..., op. cit., p. 199-206.

52. Johannes Fallati, "Der statistische Congress in Brüssel», Zeitschrift für die gesamte Staatswissenschaft /Journal of Institutional and Theoretical Economics, tome 9, 1853, p. 626-710, citation p. 87.

53. Ulrike von Hirschhausen, «People that count - The Imperial Census in Nineteenth- and Early Twentieth Century Europe and India ", in Jörn Leonhard et Ulrike von Hirschhausen [dir.], Comparing Empires... op. cit., p. 145-170; James R. Akerman [dir.], The Imperial Map..., op. cit.

54. Voir par exemple le protocole $n^{\circ} 24$ du 6 septembre 1879 dans «Further Correspondence...", loc. cit., p. 363-372. 
ce qui la mit dans l'impossibilité de définir la frontière sur la base du texte du traités ${ }^{5}$. Certes, on pouvait intégrer ces constats et expériences pratiques aux cartes, mais encore fallait-il définir avec précision les bassins hydrographiques, particulièrement fluctuants. En effet, le débit et le lit des grands fleuves évoluaient en fonction des saisons, notamment ceux du Danube et de la Drina. De ce fait, les commissions durent s'interroger sur la classification politique des îles, d'autant que le texte du traité, fondé sur la carte, prévoyait généralement que la frontière suive le thalweg. Au terme de longs débats, les différentes commissions proposèrent d'amender le texte du traité pour que la rive fasse office de frontière. Ce que les gouvernements refusèrent. On s'accorda finalement à «marquer sur la carte le thalweg existant, et, en cas de changement considérable du cours du fleuve, à laisser aux députés des provinces de chaque rive le soin de le redéfinir ${ }^{56}$.» Cette solution, adoptée en 1878 pour le Danube afin de tenir compte de l'évolution naturelle du lit du fleuve, fit ensuite figure de référence pour le tracé des frontières longeant les cours d'eau. Dès lors, les informations livrées par le texte et les cartes convergèrent avec l'expérience issue des travaux de mesure et de délimitation sur le terrain pour engendrer des représentations cartographiques. D’une part, ces documents conciliant le texte du traité et les expériences des commissions de délimitation permirent de fixer les frontières dans les Balkans. D’autre part, ils firent le lit des conflits territoriaux et des affrontements sanglants qui s'ensuivirent. Non seulement le matériel cartographique ainsi produit constitua une source d'information importante pendant la Première Guerre mondiale, mais il fut aussi utilisé durant les négociations des traités dits de la «banlieue parisienne» qui ont mis fin à la Guerre.

\section{Perspectives}

Les conflits territoriaux et le tracé des frontières sont loin d'être un enjeu spécifique au XIXe siècle. L'utilisation des cartes comme instruments politiques de domination ou de démonstration de force est un phénomène documenté depuis le XIII e siècle. Dès le XVIe siècle, on recourut à la cartographie pour construire des espaces politiques et définir des zones de domination. Enfin, à partir du XVIII siècle, les cartes jouèrent un rôle croissant dans le cadre des accords internationaux ${ }^{57}$. C'est pourquoi la territorialité évoquée par Maier et ses représentations visuelles doivent être considérées dans un contexte plus vaste, compte tenu de leurs évolutions, de leurs continuités et de leurs ruptures. Cependant - et l'exemple du Congrès de Berlin le montre

55. Wilson à Salisbury, le 12 novembre 1878, cf. "Correspondence respecting... », loc. cit., p. 58. 56. Hamley à Salisbury, le 25 septembre 1879, cf. «Further Correspondence....", loc. cit., p. 158. 57. Daniel-Erasmus Khan, Die Vertragskarte. Völkerrechtliche Untersuchung zu einem besonderen Gestaltungsmittel in der internationalen Rechtsetzung, München, C.H. Beck, 1996, p. 42-51. 
bien -, il semble que l'usage et la fonction des cartes ont évolué en ce sens au cours du XIX siècle, avec l'apparition croissante de ressources de toute sorte faisant référence au territoire et à ses caractéristiques qualitatives. La carte constituait alors un moyen de négociation, comme c'était déjà le cas au début de l'époque moderne.

Néanmoins, au XIX siècle, le consensus naissait non seulement d'un résultat présenté sous forme visuelle, mais surtout de la représentation graphique des informations, et de la méthode utilisée pour recueillir ces dernières. La production de connaissances spatiales nécessitait une expertise scientifique croissante, par exemple dans le domaine de la statistique, et, d'autre part, elle débouchait, dans des contextes souvent internationaux, sur de nouvelles formes de transfert de savoir et sur l'élaboration de normes communes. De même que la connaissance du territoire et de ses caractéristiques «intrinsèques» prit de l'importance pour le tracé des frontières, et fut intégrée aux représentations cartographiques, le rapport entre le territoire au sens matériel, qualitatif du terme - et sa représentation visuelle évolua. Aux commissions de délimitation envoyées sur le terrain par le Congrès de Berlin succédèrent d'innombrables experts qui recensèrent diverses informations sur la nature des pays, leur population et leurs ressources, afin d'en tirer des représentations graphiques mises à la disposition des gouvernements, et ce bien au-delà des Balkans. Ils finirent ainsi par élaborer les connaissances qui devaient constituer la base de toute action politique, des accords internationaux à la politique sociale. Cependant, malgré leur rôle politique déjà significatif pour les acteurs de l'époque, ces formes de création de savoir, qui comprennent de nouveaux modes de production et d'exploitation des cartes dans le sillage de la territorialisation, restent un domaine largement inexploré au sein de la recherche sur le XIX ${ }^{\mathrm{e}}$ siècle.

Ute Schneider est professeure d'histoire à l'université de Duisburg-Essen. 